\title{
Psychometric Evaluation of a 13-Point Measure of Students' Overall Competence in Community-Based Dental Education Programs
}

\author{
Vidya Ramaswamy, PhD; Wilhelm Piskorowski, DDS; Mark Fitzgerald, DDS, MS; \\ Howard A. Hamerink, DDS; Stephen Stefanac, DDS, MS; Rachel Greene, DDS; \\ Marilyn S. Lantz, PhD, DMD, MSD
}

\begin{abstract}
Since 2006, the University of Michigan School of Dentistry has used a 13-point measure of overall competence instrument to assess fourth-year dental students' end-rotation performance at community clinics. The aim of this study was to assess the reliability and validity of this instrument used by preceptors to rate students' overall competence during community-based dental education experiences. The measure was analyzed using performance ratings for all fourth-year DDS students in the graduating classes of 2012 and 2013 (combined $n=201$ ). The results were that interrater agreement was satisfactory and the measure scored high for internal consistency; also, the measure loaded highly on a single overall competence factor. Ratings on this measure did not correlate with students' final cumulative dental school GPA, but showed a significant positive correlation with their fourthyear fall patient management grades (which signify students' conscientiousness in managing patients and their families in a professional and ethical manner). There were differences in grading systems between the 2012 cohort (which used a pass/fail system) and the 2013 cohort (which used a letter grade system) and the mean ratings they received (higher for the 2013 cohort). Overall, the study found that the 13-point measure demonstrated excellent reliability and validity, suggesting it is useful in determining a student's clinical competence in these settings.
\end{abstract}

Dr. Ramaswamy is Curriculum Assessment Specialist, School of Dentistry, University of Michigan; Dr. Piskorowski is Assistant Dean for Community-Based Dental Education, School of Dentistry, University of Michigan; Dr. Fitzgerald is Associate Professor and Associate Chair, Cariology, Restorative Sciences, and Endodontics, School of Dentistry, University of Michigan; Dr. Hamerink is Adjunct Clinical Associate Professor, Biologic and Materialistic Sciences, Prosthodontic Section and Academic Program Manager for Community-Based Dental Education, School of Dentistry, University of Michigan; Dr. Stefanac is Senior Associate Dean, School of Dentistry, University of Michigan; Dr. Greene is Regional Clinical Director of Western Washington, School of Dentistry, University of Washington; and Dr. Lantz is Professor Emerita and Associate Dean for Academic Affairs Emerita, School of Dentistry, University of Michigan. Direct correspondence to Dr. Vidya Ramaswamy, School of Dentistry, University of Michigan, 1011 N. University Ave., Ann Arbor, MI 48109-1078; 734-647-4218; ramaswav@umich.edu.

Keywords: dental education, community-based dental education, community rotations, assessment, clinical competence

Submitted for publication 11/23/15; accepted 3/29/16

$\mathrm{P}$ revious studies have found that communitybased dental education (CBDE) experiences have a positive impact on student learning. ${ }^{1-5}$ The incorporation of CBDE into predoctoral dental curricula offers three significant advantages in facilitating the development of competent oral health professionals. First, it gives students an opportunity to develop their competence in providing patientcentered care in public health settings that are closer to their future professional practice than in the school clinic. ${ }^{3}$ A second advantage of CBDE is that it provides for students to be assessed at the "does" level in Miller's pyramid - a hierarchical framework in which levels of clinical competence are defined as "knows," "knows how," "shows how," and "does." This opportunity is important because higher level assessments such as the objective structured clinical examination (OSCE) used in dental schools typically do not go beyond the "shows how" level in Miller's pyramid. A third advantage is that CBDE allows educators to perform longitudinal assessments of students' overall performance, thus adding to the sources of data schools can use in multisource assessments of student competence. ${ }^{7}$ 
The students' experience in CBDE settings is critical because it reflects their ability to demonstrate competence independent of curricular settings and supervision by the regular dental school faculty. As Albino et al. noted, "The prevailing recommendation for measuring general (overall) competence is a pre-graduation internship of at least two months' duration that resembles the work environment, tasks, and responsibilities of entry-level practitioners." Albino et al. qualified this recommendation by suggesting that, for coaching consistency, students should work under the daily supervision of a small group of faculty members who assess the students' ability to repeatedly demonstrate competence, to perform a seamless transition between individual competencies during patient care, and to show a depth of knowledge and perform various self-management and professionalism competencies such as punctuality, decorum, appearance, stress management, and capacity for self-assessment and self-correction. Miller argued that "the collective wisdom of faculty members who have consistent opportunities to observe and interact with the student is the essential core of performance assessment. ${ }^{16}$ According to Albino et al., that point has been endorsed by virtually every review of assessment best practices in health professions education. ${ }^{8}$

The emphasis on assessing overall competence has increased in dental education. The Commission on Dental Accreditation (CODA) standards implemented in 2013 state that dental education programs "should assess overall competence, not simply individual competencies, in order to measure the graduate's readiness to enter the practice of general dentistry" (intent statement for Standard 2-23). ${ }^{9}$ Not surprisingly, interest in identifying valuable measurement tools to assess dental students' overall competence has also increased, adding to the need to develop psychometrically sound assessments based on a conceptual framework in CBDE settings. ${ }^{10}$

Our literature review, however, suggested a lack of validated tools to assess student performance at community sites. Additionally, we believe that using for CBDE the same assessment instruments used at dental schools would be both burdensome and inappropriate because the information we seek about students' performance at community sites is quite different from the "in-training" performance data collected at the school. Instruments used at our school to assess student competence (for example, in scaling and root planing, diagnosis and treatment planning, placement of composite restorations) are developed by the departments and measure what we refer to as "silo" competencies: procedural skills in single, distinctive aspects of performance, which comprise a significant proportion of the third- and fourth-year comprehensive care grades. At the community clinics, we are not interested in measuring those types of competencies; rather, we want to measure students' ability to "put it all together" during the delivery of patient care in a private practice environment in which they are performing various types/aspects of care in a single clinic session.

Another form of assessment used is cumulative GPAs, which include basic, behavioral, and clinical didactic course and clinic grades. In addition, the fourth-year fall patient management grade is an average of many assessments of performance of the same skill or attitude over time, related to respect, responsibility, communication skills, integrity, and critical thinking. While valuable in assessing students' overall abilities, GPAs and patient management grades are not able to capture the distinctive nature of learning in CBDE.

Beginning in the fall term 2006, we implemented a 13-point measure of overall competence (13-point MOC) instrument for use by preceptors at community-based clinics as a performance measure in the University of Michigan School of Dentistry's CBDE program. The 13-point MOC is intended for use as a global assessment of students' performance while on rotation at a community site. It allows for ratings by multiple preceptors over a period of time. The aim of this study was to determine the reliability and validity of the 13-point MOC for use in the outreach clinic setting as a measure of students' overall competence.

\section{Methods}

This study was determined to be exempt from oversight by the Institutional Review Board for the Behavioral and Health Sciences at the University of Michigan (HUM00107190). The 13-point MOC form was developed collaboratively by the school's associate dean for patient services, associate dean for academic affairs, director of outreach, and the comprehensive care clinic directors in consultation with CBDE clinic staff dentists. (The form is available from the corresponding author.) Performance dimensions for the MOC were derived from a literature review, feedback from school clinic directors and CBDE preceptors, and analysis of student 
performance on clinical test cases. A major focus in creating the measure was selecting items focused on critical thinking skills (for example, diagnosis and treatment planning, analysis of dental/medical history) and items that capture students' ability to function in an environment that closely resembles what they will experience in their post-graduation clinical practice (following clinical protocols, communication and verbal skills, level of independence, and self-assessment and patient management skills, in addition to clinical knowledge and technical ability). All performance dimensions included in the 13-point MOC mapped to competency statements in the school's competencies for the new dental graduate.

Community clinic staff dentists serve as adjunct faculty preceptors for students on rotation. They complete a rating of student performance at the end of each rotation using the 13-point MOC on a five-point scale from $1=$ does not meet expectations to $5=$ exceeds expectations. They are also invited to provide written comments about the student's performance. Each student is given a copy of his or her 13-point MOC assessment and must sign it to indicate it was received. Students also provide assessments of their experience at each site.

Although, in developing the 13-point MOC, we sought to identify the dimensions in which students would receive feedback, we did not try to anchor them in the same performance criteria used at the dental school. Rather, we sought to provide preceptors with a tool that would allow them to provide feedback to students that they thought critical for effective performance in the community clinic environment. This process was facilitated in several ways. The associate dean for academic affairs provided feedback about use of the 13-point MOC and reviewed analyses of the outcomes data derived from its use and any actions taken by the program based on these analyses at annual retreats attended by representatives of all participating community clinics. Moreover, the directors of the CBDE program provide ongoing one-on-one orientations about the use and goals of the MOC to all new community clinic staff dentists who serve as adjunct faculty preceptors.

\section{Rotation Sites}

Data for 2012 and 2013 were used for this study. In those (and other) years, dental students in the fourth year (D4) participated in rotations in community settings, and the preceptors rated the students' performance on the rotations using the 13-point MOC. D4 students were scheduled for five two-week rotations scheduled every two months. Each rotation was a minimum of four days per week with the majority being five days per week. Students rotated through various health care models in their rotations. Rotation sites included Federally Qualified Health Centers (FQHCs), private practice settings, Indian Health Service clinics, and community dental centers. The number of preceptors ranged from one to 16 across all sites, with two being the average. The number of students at each site ranged from one to eight, with most sites typically having one or two students for each rotation. The first evaluation for each student was used for the data analysis to ensure a consistent format for data selection. Approximately half of the ratings were part of rotation one for the data analysis used in this study (Table 1).

All preceptors were provided with a training packet outlining school protocols, student manuals, and resource links. The course directors for CBDE did an in-person initial training and orientation in an individual or group setting. Training included an overview of the rubric parameters with the help of illustrative exercises. Training was repeated yearly and at least one additional time during the first year of preceptor activity. Interactions between a preceptor and students were mentored by one of the course directors during the first day or two of the preceptor's activity. Each preceptor had access to a member of

Table 1. Students in each community-based dental education rotation cycle by years in study

\begin{tabular}{cccc} 
Rotation Number & $2012(\mathrm{n}=106)$ & $2013(\mathrm{n}=95)$ & Total $(\mathrm{n}=201)$ \\
\hline 1 & $46(43 \%)$ & $49(52 \%)$ & $95(47 \%)$ \\
2 & $27(26 \%)$ & $16(17 \%)$ & $43(21 \%)$ \\
3 & $18(17 \%)$ & $17(18 \%)$ & $35(17 \%)$ \\
4 & $8(8 \%)$ & $7(7 \%)$ & $15(8 \%)$ \\
5 & $7(7 \%)$ & $6(6 \%)$ & $13(7 \%)$
\end{tabular}

Note: Percentages may not total $100 \%$ because of rounding. 
the CBDE team at any time. The course directors monitored postings daily and flagged any aberrant scores for follow-up. Summation reports allowed comparison of scores of all preceptors.

\section{Data Analysis}

Interrater agreement (reliability) on the 13-point MOC was measured by the percentage of times two raters had the same rating for the same student performance either at the same site or at a different site for the 2012 cohort. Interrater agreement is considered good when many ratees receive the same rating for the same performance. ${ }^{11}$

The two groups' scores were compared on the 13-point MOC to assess its validity. Data on the 13-point MOC were examined to see if they allowed for factor analysis using the following criteria: significant sphericity, skewness $>2$, kurtosis $<7$, eigenvalues $>1.5$, and at least three variables with a loading $>0.40$ for each factor. ${ }^{12,13}$ Analyses used were KMO and Bartlett's test of sphericity. Exploratory factor analysis was used to investigate the internal structure of the 13-point MOC. A principal component analysis with eigenvalues criterion $>1.5$ and factor loading was used to extract factors.

To assess discriminant and concurrent validity, students' total scores on the 13-point MOC were analyzed for correlation with their final cumulative GPA and patient management (comprehensive care) grades for the fall term of their D4 year. The final cumulative GPA served as an indicator of students' overall dental school performance, whereas the patient management grades were an indicator of students' conscientiousness in managing patients and their families in an ethical and professional manner, therefore relating specifically to clinical practice. Patient management grades were assigned by patient care coordinators in the dental school clinics who monitor students' professionalism, number and frequency of appointments, progress in treatment planning, and case completions on a daily basis. Patient care coordinators acted as a liaison among faculty, students, and patients and worked closely with students in their patient treatment activity. Patient management grades were assigned for the 2012 cohort using a pass/fail system; for the 2013 cohort, a scaled grading system with letter grades was used. All data were analyzed using SPSS, Version 22 (Chicago, IL, USA).

\section{Results}

The analyses were conducted with performance ratings for all fourth-year DDS students in the graduating classes of $2012(\mathrm{n}=106)$ and $2013(\mathrm{n}=95$; combined $n=201$ ). In our analysis of interrater agreement (reliability), most students received a high score of 4 or 5 . The range for complete agreement was from $40 \%$ ("treatment planning ability" and "ability to selfevaluate") to 54\% ("follows clinical protocols"). Raters tended to be within a point of each other most of the time ( $81 \%$ to $95 \%)$ for all 13 items (Table 2). The Cronbach's alpha for the 13-point MOC was 0.96, indicating that it was highly reliable (i.e., internally consistent). The item total correlations were all high and ranged from 0.75 to 0.86 (Table 3).

Table 2. Interrater agreement on items on 13-point measure of overall competence for students in 2012

\begin{tabular}{lcc} 
Item & $\begin{array}{c}\text { Percentage of Times } \\
\text { Two Raters Had Same Scores for } \\
\text { Same Student Across Sites }\end{array}$ & $\begin{array}{c}\text { Percentage of Times } \\
\text { Raters Had Scores Within } \\
\text { One Point of Each Other }\end{array}$ \\
\hline 1. Follows clinic protocols & $54 \%$ & $90 \%$ \\
2. Analysis of dental/medical history & $43 \%$ & $95 \%$ \\
3. Knowledge of prescribing oral medications & $47 \%$ & $91 \%$ \\
4. Caries detection ability & $43 \%$ & $83 \%$ \\
5. Overall diagnostic skills & $41 \%$ & $86 \%$ \\
6. Patient management skills & $41 \%$ & $89 \%$ \\
7. Treatment planning ability & $40 \%$ & $81 \%$ \\
8. Communication/verbal skills & $45 \%$ & $90 \%$ \\
9. Technical ability & $43 \%$ & $90 \%$ \\
10. Clinical knowledge & $45 \%$ & $88 \%$ \\
11. Level of independence & $49 \%$ & $94 \%$ \\
12. Ability to self-evaluate & $40 \%$ & $90 \%$ \\
13. Quality of interaction with staff and dentists & $52 \%$ & $89 \%$
\end{tabular}


In the assessment of validity, the KMO and Bartlett's test of sphericity was significant $(p<0.001)$. The skewness of all items was less than 2.00, and kurtosis of all items was less than 5. Factor loadings were high (greater than 0.76 for all items) and showed the presence of one principal factor (overall competence), which was confirmed by the scree plot. This factor accounted for $70 \%$ of variance in the scores. Students performed very well on all the 13 dimensions of the MOC (Table 4), with mean scores ranging from $4.12(\mathrm{SD}=0.81)$ for "caries detection ability" to $4.54(\mathrm{SD}=0.72)$ for "quality of interaction with staff and dentists."

The results showed no correlations between the students' MOC total scores and final cumulative GPA (Table 5). However, they showed a low, positive, and significant correlation between the MOC scores and patient management grades for the fall term of the D4 year. When we compared the scores of the 2012 and 2013 cohorts, there were significant differences

Table 3. Exploratory factor analysis and item total correlation for items on the 13-point measure of overall competence $(\mathrm{n}=\mathbf{2 0 1})$

\begin{tabular}{lcc} 
Item & Factor 1 & Item Total Corre \\
\hline 1. Follows clinic protocols & 0.76 & $0.78^{* *}$ \\
2. Analysis of dental/medical history & 0.87 & $0.84^{* *}$ \\
3. Knowledge of prescribing oral medications & 0.88 & $0.86^{* *}$ \\
4. Caries detection ability & 0.82 & $0.82^{* *}$ \\
5. Overall diagnostic skills & 0.88 & $0.85^{* *}$ \\
6. Patient management skills & 0.82 & $0.82^{* *}$ \\
7. Treatment planning ability & 0.85 & $0.83^{* *}$ \\
8. Communication/verbal skills & 0.83 & $0.82^{*}$ \\
9. Technical ability & 0.78 & $0.75^{* *}$ \\
10. Clinical knowledge & 0.86 & $0.81^{* *}$ \\
11. Level of independence & 0.82 & $0.77^{* *}$ \\
12. Ability to self-evaluate & 0.89 & $0.86^{* *}$ \\
13. Quality of interaction with staff and dentists & 0.79 & $0.77^{* *}$
\end{tabular}

Note: Factor one accounted for $70 \%$ of variance in scores; Cronbach's alpha was 0.96.

*Significant at $\mathrm{p}<0.05 ;{ }^{* *}$ significant at $\mathrm{p}<0.01$

Table 4. Descriptive statistics for each item on the 13-point measure of overall competence and Cronbach's alpha $(\mathrm{n}=\mathbf{2 0 1})$

\begin{tabular}{|c|c|c|c|c|c|c|}
\hline Item & $\begin{array}{l}\text { es Not Meet } \\
\text { spectations } \\
1 \\
\text { N (\%) }\end{array}$ & $\begin{array}{c}2 \\
N(\%)\end{array}$ & $\begin{array}{c}\text { Meets } \\
\text { Expectations } \\
3 \\
\mathrm{~N}(\%)\end{array}$ & $\begin{array}{c}4 \\
\text { N (\%) }\end{array}$ & $\begin{array}{c}\text { Exceeds } \\
\text { Expectations } \\
5 \\
\mathrm{~N}(\%)\end{array}$ & Mean (SD) \\
\hline 1. Follows clinic protocols & $2(1 \%)$ & $6(3 \%)$ & $7(4 \%)$ & $83(42 \%)$ & $102(5$ & 4.3 \\
\hline 2. Analysis of dental/medical history & 0 & $1(1 \%)$ & $15(8 \%)$ & $93(46 \%)$ & $92(46 \%)$ & $4.37(0.64)$ \\
\hline 3. Knowledge of prescribing oral medications & 0 & $2(1 \%)$ & $13(7 \%)$ & $94(50 \%)$ & $78(42 \%)$ & $4.33(0.65)$ \\
\hline 4. Caries detection ability & $1(1 \%)$ & $5(3 \%)$ & $33(17 \%)$ & $87(44 \%)$ & $70(36 \%)$ & $4.12(0.81)$ \\
\hline 5. Overall diagnostic skills & 0 & $2(1 \%)$ & $28(14 \%)$ & $97(48 \%)$ & $74(37 \%)$ & $4.21(0.71)$ \\
\hline 6. Patient management skills & 0 & $1(1 \%)$ & $16(8 \%)$ & $78(39 \%)$ & $105(53 \%)$ & $4.44(0.66)$ \\
\hline 7. Treatment planning ability & 0 & $1(1 \%)$ & $37(19 \%)$ & $71(37 \%)$ & $82(43 \%)$ & $4.23(0.77)$ \\
\hline 8. Communication/verbal skills & $1(1 \%)$ & $2(1 \%)$ & $12(6 \%)$ & $75(37 \%)$ & $111(55 \%)$ & $4.46(0.70)$ \\
\hline 9. Technical ability & $1(1 \%)$ & 0 & $28(14 \%)$ & $111(55 \%)$ & $61(30 \%)$ & $4.15(0.68)$ \\
\hline 10. Clinical knowledge & 0 & $1(1 \%)$ & $26(13 \%)$ & $90(45 \%)$ & $84(42 \%)$ & $4.28(0.70)$ \\
\hline 11. Level of independence & 0 & 0 & $20(10 \%)$ & $85(42 \%)$ & $96(48 \%)$ & $4.38(0.66)$ \\
\hline 12. Ability to self-evaluate & 0 & $4(2 \%)$ & $17(9 \%)$ & $87(44 \%)$ & $92(46 \%)$ & $4.34(0.72)$ \\
\hline 13. Quality of interaction with staff and dentists & $1(1 \%)$ & $4(2 \%)$ & $9(5 \%)$ & $58(29 \%)$ & $128(64 \%)$ & $4.54(0.72)$ \\
\hline
\end{tabular}

Note: Responses were provided on a five-point scale from $1=$ does not meet expectations to $3=$ meets expectations to $5=$ exceeds expectations. 
Table 5. Correlations among students' 13-point measure of overall competence (MOC) scores, cumulative GPA, and fourth-year fall patient management grades (PMG)

\begin{tabular}{lccc} 
Measure & $\begin{array}{c}\text { 13-Point } \\
\text { MOC }\end{array}$ & $\begin{array}{c}\text { Cumulative } \\
\text { GPA }\end{array}$ & PMG \\
\hline 13-point MOC & & -0.03 & $0.15^{*}$ \\
Cumulative GPA & & & $0.30^{* *}$
\end{tabular}

*Significant at $\mathrm{p}<0.05 ; * *$ significant at $\mathrm{p}<0.01$

between the two cohorts in their total scores as well as on some of the items, with the 2013 cohort having higher scores than the 2012 cohort (Table 6).

\section{Discussion}

The ability of graduating dental students to perform well in real-world settings is not only an important indicator of overall competence; it is required for independent practice. The CBDE experience serves as a capstone learning experience before students move on to independent practice. The 13-point MOC can also be used by practicing staff dentists to rate students' performance by direct observation of their functioning in a real-world environment, in which students are performing at the "does" level of Miller's pyramid. ${ }^{6}$ Clinical test cases and OSCEs administered as competency assessments measure student performance at the "shows how" level, and this performance occurs in a controlled setting. ${ }^{8}$ Therefore, the 13-point MOC contributes to our competency assessment armamentarium by providing an assessment of specific dimensions of competence and overall competence at the "does" level.

The 13-point MOC measures a single "overall competence" factor as reflected in the exploratory factor analysis, which found that all 13 items loaded highly $(0.76$ to 0.89$)$ on this factor. This factor accounted for $70 \%$ of variance in the scores. These findings were corroborated in the high item total correlations ( 0.75 to 0.86$)$. The 13 items in the MOC tool provide a comprehensive measure of clinical competence that includes ability to follow clinic protocols, analysis of patient's history, ability to detect caries, diagnostic skills, patient management skills, technical ability, clinical knowledge, knowledge of prescribing medicines, diagnostic skills, treatment planning, communication, self-assessment, and quality of interaction with staff and dentists, all of which support its use as a measure of overall competence. The lack of correlation we found with final cumulative GPA and a weak but significant positive correlation with patient management grades suggest it is measuring a construct that is not school-dependent but relates instead to trusting students with patient care and management. It is important to have such performance assessments available for use in external rotation settings, which

Table 6. Descriptive statistics on the 13-point measure of overall competence for 2012 and 2013 cohorts

\begin{tabular}{|c|c|c|c|}
\hline Item & $\begin{array}{c}2012 \\
(n=106) \\
\text { Mean (SD) }\end{array}$ & $\begin{array}{c}2013 \\
(\mathrm{n}=95) \\
\text { Mean (SD) }\end{array}$ & $\begin{array}{c}\text { T value } \\
\text { (sig) }\end{array}$ \\
\hline 1. Follows clinic protocols & $4.30(0.82)$ & $4.48(0.73)$ & 1.61 \\
\hline 2. Analysis of dental/medical history & $4.29(0.62)$ & $4.46(0.67)$ & 1.89 \\
\hline 3. Knowledge of prescribing oral medications & $4.16(0.70)$ & $4.51(0.55)$ & $3.79^{* *}$ \\
\hline 4. Caries detection ability & $3.94(0.80)$ & $4.32(0.79)$ & $3.31^{* *}$ \\
\hline 5. Overall diagnostic skills & $4.07(0.71)$ & $4.37(0.68)$ & $3.07^{* *}$ \\
\hline 6. Patient management skills & $4.36(0.73)$ & $4.52(0.56)$ & 1.77 \\
\hline 7. Treatment planning ability & $4.00(0.81)$ & $4.46(0.65)$ & $4.32^{* *}$ \\
\hline 8. Communication/verbal skills & $4.40(0.79)$ & $4.53(0.58)$ & 1.34 \\
\hline 9. Technical ability & $3.97(0.74)$ & $4.35(0.56)$ & $4.03^{* *}$ \\
\hline 10. Clinical knowledge & $4.14(0.74)$ & $4.43(0.63)$ & $2.98^{* *}$ \\
\hline 11. Level of independence & $4.31(0.68)$ & $4.45(0.63)$ & 1.52 \\
\hline 12. Ability to self-evaluate & $4.21(0.79)$ & $4.47(0.60)$ & $2.64^{*}$ \\
\hline 13. Quality of interaction with staff and dentists & $4.51(0.84)$ & $4.57(0.56)$ & 0.65 \\
\hline Total & $53.86(7.67)$ & $57.36(7.26)$ & $3.31^{* *}$ \\
\hline
\end{tabular}

Note: Responses were provided on a five-point scale from $1=$ does not meet expectations to $3=$ meets expectations to $5=$ exceeds expectations.

*Significant at $\mathrm{p}<0.05 ;{ }^{* *}$ significant at $\mathrm{p}<0.01$ 
more closely approximate independent practice than most dental school clinic experiences.

The psychometric analysis of the 13-point MOC suggested it is a reliable and valid tool. It showed excellent reliability (0.96), indicating the tool had high internal consistency. Interrater agreement was excellent, with raters within one point of each other more than $80 \%$ of the time. This finding makes the tool practically useful as it is used across varied outreach settings by different raters.

The 13-point MOC also captured differences between the 2013 cohort (who received letter grades in comprehensive care) and the 2012 cohort (who received pass/fail grades in comprehensive care), with the 2013 cohort receiving higher scores than the 2012 cohort. One hypothesis accounting for this outcome is that students receiving a letter grade are more motivated to perform well. If that is the case, then in addition to reflecting the measure's ability to discriminate, it may fit well with the school's letter grade system. Almost all students either met or exceeded the performance expectations of the outreach preceptors, indicating that the preceptors were satisfied with their students and considered them competent. This is important for a successful CBDE program that includes many $\mathrm{FQHC}$ sites.

Finally, the outcomes of the 13-point MOC provide the dental school with important information about its educational program. We tally annually the performance ratings of all students across each dimension of the MOC, and if more than $5 \%$ of the student ratings in any dimension assessed by the MOC fall into the "does not meet expectations/needs improvement" range (score of 1 or 2), we investigate the matter at a programmatic level. For example, for the Class of 2007 (the first class for which the MOC was used), we found more than $6 \%$ of the ratings in the dimension of "level of independence" fell into the 1-2 range. Upon further investigation, we found that most of the independence concerns were related to performance of oral surgery procedures. In our curriculum at that time, the students had rotations in oral surgery during the D3 year. Some students had the rotation early in the D3 year and had no other oral surgery experience after the rotation, so they may have been arriving at the CBDE sites unprepared to perform exodontia. After discussions with comprehensive care directors and the Department of Oral Surgery, it was decided that we would implement a D4 oral surgery rotation. In the years that followed, the numbers of 1-2 ratings on "level of independence" dropped sharply and were zero in 2012 and 2013 (Table 5).
Validating scores on the 13-point MOC against other measures of actual performance in clinical settings and replicating the measure in other outreach settings will provide additional valuable information about the usefulness of this scale. One possible limitation to our study was the effect of students' high scores on this tool. This was most likely due to the fact that we were measuring criterion-level performance in students who were close to graduation. To provide for more variation in scores, the scale could potentially be expanded to a 7-point scale with clear criteria defining each point on the scale. We also suggest adding a global rating score to this tool in addition to the score on the 13 components, as this would help in obtaining an overall rating for each student that would serve as an additional point of information and strengthen any psychometric analysis. Another future possibility would be to add more items to this tool in the domains of ethics and professionalism to more clearly capture additional important elements of competent performance in these domains. Finally, since the study was conducted at only one dental school, its results may not be generalizable for students at other dental schools.

\section{Conclusion}

This study found that the 13-point MOC is a valid and reliable tool that can effectively measure students' competence in CBDE settings. It provides a distinctive assessment of students' performance in external clinical rotations that are not captured in traditional measures such as GPAs. It can also serve as a capstone assessment of the graduating dental student's readiness to practice and adds to the quality of assessment by preceptors in community settings. Further validation of assessment tools used during CBDE rotations will provide even more useful information about this measure.

\section{REFERENCES}

1. Nayar P, McFarland K, Lange B, et al. Supervising dentists' perspectives on the effectiveness of communitybased dental education J Dent Educ 2014;78(8):1139-44.

2. DeCastro JE, Bolger D, Feldman CA. Clinical competence of graduates of community-based and traditional curricula. J Dent Educ 2005;69(12):1324-31.

3. Strauss RP, Stein MB, Edwards J, Nies KC. The impact of community-based dental education on students. J Dent Educ 2010;74(10 Suppl):S42-55.

4. Smith M, Lennon MA, Brook AH, Robinson PG. A randomized controlled trial of outreach placement's ef- 
fect on dental students' clinical confidence. J Dent Educ 2006;70(5):566-70.

5. Smith M, Lennon MA, Brook AH, et al. A randomized controlled trial of the effect of outreach placement on treatment planning by dental students. Br Dent J 2006;Suppl:27-31.

6. Miller GE. The assessment of clinical skills/competence/ performance. Acad Med 1990;65(9):63-7.

7. Moore U, Dunham J. Issues with assessing competence in undergraduate dental education. Eur J Dent Educ 2011;15:53-7.

8. Albino JEN, Young SK, Neumann LM, et al. Assessing dental students' competence: best practice recommendations in the performance assessment literature and investigation of current practices in predoctoral dental education. J Dent Educ 2008;72(12):1405-35.

9. Commission on Dental Accreditation. Accreditation standards for dental education programs. At: www.ada.org/ /
media/CODA/Files/predoc 2013.ashx. Accessed 14 Nov. 2014.

10. Khatami S, MacEntee MI. Evolution of clinical reasoning in dental education. J Dent Educ 2011;75(3):321-8.

11. Graham M, Milanowski A, Miller J. Measuring and promoting interrater agreement of teacher and principal performance settings. Center for Educator Compensation Reform. 2012. At: files.eric.ed.gov/fulltext/ED532068. pdf. Accessed 5 Dec. 2014.

12. Schönrock-Adema J, Heijne-Penninga M, Van Hell EA, Cohen-Schotanus J. Necessary steps in factor analysis: enhancing validation studies of educational instrumentsthe PHEEM applied to clerks as an example. Med Teach 2009;31(6):e226-32.

13. Kossioni AE, Lyrakos G, Ntinalexi I, et al. The development and validation of a questionnaire to measure the clinical learning environment for undergraduate dental students (DECLEI). Eur J Dent Educ 2013;18(2):71-9. 